\title{
Antibody research targeting Cathepsin S for cancer therapy
}

\author{
Hang Fai Kwok ${ }^{1,2,3}$ \\ ${ }^{1}$ Department of Oncology, University of Cambridge, Cambridge, UK \\ ${ }^{2}$ School of Pharmacy, Queen's University Belfast, Belfast, UK \\ ${ }^{3}$ Faculty of Health Sciences, University of Macau, Macau, China \\ Email: hfk24@cam.ac.uk
}

Received January $14^{\text {th }}, 2013$; revised March $10^{\text {th }}, 2013$; accepted April $13^{\text {th }}, 2013$

Copyright (C) 2013 Hang Fai Kwok. This is an open access article distributed under the Creative Commons Attribution License, which permits unrestricted use, distribution, and reproduction in any medium, provided the original work is properly cited.

\begin{abstract}
Cathepsin $S$ is a cysteine protease highly expressed in many type of cancers including colorectal, prostate, breast, and glioblastoma's. It is involved in tumor progression through extracellular matrix remodeling. In recent years, antibody research specifically targeting Cathepsin S to block/inhibit tumorigenic effects were generated some positive preclinical data. This antagonistic antibody was demonstrating efficacy in multiple in vitro/in vivo cancer models both as a monotherapy and in combination with approved agents. This mini-review provides an overview of therapeutic antibody targeting Cathepsin S strategies in the last half decade, focusing on the rationale of cell-surface Cathep$\sin \mathrm{S}$ targeted and their potential clinical application.
\end{abstract}

Keywords: Cathepsin S; Tumor; Therapeutic Antibody

\section{INTRODUCTION}

In the past 10 - 20 years, molecularly targeted cancer therapies aim to inhibit the activity of an up-regulated oncoprotein in an attempt to revert the evolved proliferative/survival advantage it provides the tumor. Cathepsin $S$ is a proteolytic enzyme and represents potential therapeutic targets in human cancers. It predominantly functions as endopeptidases within endolysosomal vesicles of healthy cells, and involved in many physiological processes such as differentiation, protein turnover, degradation and apoptosis. In various types of malignancies, Cathepsin S has been reported in associate with tumor progression and metastasis. In the last decade, more and more solid evidence and direct proofs demonstrate that Cathepsin S is highly overexpressed/up-regulated in many cancer cell lines and contributes to the development and progression of the cancer phenotype.
Nowadays, the most specific targeted therapies currently in use are monoclonal antibodies. Therefore, in recent years, scientists started to investigate the potential to target Cathepsin $\mathrm{S}$ using an antagonistic monoclonal antibody specifically block/inhibit these tumorigenic effects such as tumor invasion and angiogenesis. In this article, we outline the roles of a specific antagonistic antibody targeting Cathepsin $\mathrm{S}$ in the tumor microenvironment, and then we further focus on its therapeutic potential for the treatment of cancer as a monotherapy and in combination with approved agents.

\section{CELL SURFACE ASSOCIATED CATHEPSIN S FOR ANTIBODY TARGETING}

Cathepsin S distinguishes itself from many other family members with a very restricted normal tissue expression, found mainly in cells of monocyte/macrophage lineage and lymphatic tissue. It acts as a key role in MHC class II presentation through invariant chain degradation [1,2]. Cathepsin S has been found to be up-regulated and associated with disease aggressiveness in a number of tumor types [3-5] and is of independent prognostic value in glioblastoma [6]. The pilot retrospective study of prevalence of Cathepsin S expression in primary and metastatic colorectal cancer patients has demonstrated that greater than $95 \%$ of tumors had detectable Cathepsin S expression with significantly increased staining in tumors compared with matched normal colon [7]. The expression of Cathepsin S on the surface of pancreatic and colon carcinoma cells has also been revealed recently through how cell surface associated Cathepsin S can be targeted to mediate ADCC by a fully humanized Cathepsin S antibody. Cathepsin S was expressed on the surface of tumor cells representative of colorectal and pancreatic cancer with $23 \%$ - $79 \%$ positive expression [8]. Furthermore the binding of the Cathepsin S antibody to sur- 
face associated Cathepsin S resulted in natural killer (NK) cell targeted tumor killing. In a colorectal cancer model the Cathepsin S antibody elicited a 22\% cytotoxic effect [8]. The demonstration of Cathepsin S targeted ADCC efficacy opens up the possibility of targeting other enzymes such as MMP's and ADAMs in a similar way.

\section{ANTIBODY-MEDIATED INHIBITION OF CATHEPSIN S BLOCKS TUMOR INVASION}

In recent years, a panel of monoclonal antibodies was raised to human Cathepsin S [9]. The effects of a selected antibody were successfully determined by cellbased invasion and proteolysis assays. Endothelial cell tube formation and aorta sprouting assays were also performed to examine antiangiogenic effects. In vivo effects were also evaluated using HCT116 xenograft studies. The results from this selected Cathepsin S inhibitory antibody significantly blocked invasion of a range of cancer cell lines through inhibition of extracellular Cathepsin S-mediated proteolysis. Researchers also subsequently demonstrated the enhancement of expression of Cathepsin S in colorectal adenocarcinoma biopsies when compared with normal colon tissue. Furthermore, the Cathepsin S inhibitory antibody blocked endothelial cell capillary tube formation and aortic microvascular sprouting. The administration of the Cathepsin S inhibitory antibody resulted in inhibition of tumor growth and neovascularization of HCT116 xenograft tumors [9].

This application of an antibody inhibitor to Cathepsin $S$ represents a new modality for treatment of cancer that not only inhibits the invasion of tumors, but also targets neovascularization. Moreover, its selectivity to Cathepsin $\mathrm{S}$ and preferential targeting to extracellular pathologic Cathepsin S may circumvent off-target effects. The Cathepsin S inhibitory antibody could have clinical utility in the treatment of colorectal and other invasive tumors in which elevated levels of Cathepsin S have been identified. The combination of chemotherapy treatments with an anti-invasive agent such as the Cathepsin $\mathrm{S}$ antagonistic antibody could lead to improved clinical outcomes in aggressive and metastatic tumors.

\section{ANTIBODY TARGETING OF CATHEPSIN S INHIBITS ANGIOGENESIS \& SYNERGISTICALLY ENHANCES ANTI-VEGF}

Cathepsin $\mathrm{S}$ has been shown to play an important role in angiogenesis as has vascular endothelial growth factor (VEGF). Further studies have shown that Cathepsin S is significantly overexpressed by endothelial cells during tumor angiogenesis $[10,11]$ and compellingly Cathepsin $\mathrm{S}$ knockout mice had a significant reduction in tumor- associated angiogenic switching and neovascularization [12]. Angiogenesis is a key hallmark of tumorigenesis and its inhibition is a proven strategy for the development of novel anti-cancer therapeutics. Therefore, in recent years, researchers sought to assess the anti-angiogenic effect of Cathepsin S inhibitory antibody when combined with anti-VEGF on vascular development.

Cathepsin S promotes pericellular hydrolysis of extracellular matrix components in the tumor microenvironment and facilitates endothelial invasion [13]. The Cathepsin S inhibitory antibody blocks extracellular proteolysis, inhibiting endothelial invasion and tube formation in cell-based assays. The anti-angiogenic effects of Cathepsin S inhibitory antibody were also shown in vivo where it significantly retarded the development of vasculature in human xenograft models. Furthermore, when the Cathepsin S inhibitory antibody was combined with an anti-VEGF antibody, a synergistic inhibition of microvascular development was observed [13]. Taken together, the finding clearly demonstrates that the antibody-mediated targeting of Cathepsin S paves a new way of inhibiting angiogenesis. In addition, when used in combination with anti-VEGF therapies, the Cathepsin S inhibitory antibody has the potential to significantly enhance current treatments of tumor neovascularization and may also be of use in the treatment of other conditions associated with inappropriate angiogenesis.

\section{INHIBITION OF CATHEPSIN S BY ITS INHIBITORY ANTIBODY ENCHANCES THE EFFICACY OF CANCER CHEMOTHERAPY}

As mentioned previously, the development and treatment of Cathepsin S specific inhibitory antibody with different type of cancer cell lines significantly attenuated tumor cell invasion, mediated through the ability of blocking Cathepsin S cleavage of ECM proteins. Moreover, the inhibition of Cathepsin S using its specific inhibitory antibody attenuated growth and vascularization of colorectal xenograft tumors $[9,13]$. The combined anti-invasive and anti-angiogenic effects of the Cathepsin S specific inhibitory antibody may be a useful therapeutic approach, especially when used in combination with conventional chemotherapy. In order to answer this question, researchers further investigated whether Cathepsin S expression levels were affected by chemotherapy in human cancer cell lines using RT-PCR and colorectal xenograft models to analyze the effects of the combination therapy (Cathepsin S inhibitory antibody + CPT11) on tumor progression and on tumor vascularization by immunohistochemical staining of tumors. The results showed that chemotherapy can actually increase the expression of Cathepsin S and that when administered as a combination regimen, the Cathepsin S inhibitory antibody sig- 
nificantly enhanced the efficacy of CPT-11 [14]. Collectively, these observations clearly show that Cathepsin S inhibitory antibody has the potential to be combined with existing therapeutic strategies for the treatment of cancer. However, taken together with the observation that chemotherapies may actually increase the expression of the pro-invasive and pro-angiogenic Cathepsin $\mathrm{S}$ in the tumor microenvironment, it highlights a clear rationale for the co-application of a specific inhibitor such as Cathepsin S inhibitory antibody to reduce post-treatment tumor re-growth.

\section{PERSPECTIVE}

The principal challenge of anti-cancer therapy is to specifically inhibit the malignant activity of tumors whilst leaving normal and healthy cells unaffected [15]. The advent of monoclonal antibody technology has revolutionized the pharmacological capacity to both specifically block protein-protein interactions [16] and deliver cytotoxic events to a tumor-specific antigen [17]. The combination of high affinity, long in vivo half-life and low background toxicity has made human monoclonal antibody a useful anti-cancer tool [18]. Whilst there are also small molecule approaches to specifically inhibiting a protein drug target, the large antibody-antigen binding surface provided by a monoclonal antibody approach becomes critical when targeting an individual member of a family of structurally related proteins such as Cathepsin family.

The evidence available to date indicates that the use of an antibody that can selectively antagonize Cathepsin S in the tumor milieu may help circumvent toxicity issues that are likely to arise with small molecule inhibitors. Further in vivo stability study on the humanized Cathepsin S inhibitory antibody showed that this antibody possesses a stable half-life of 274 hours, and has the ability to target cell surface associated Cathepsin S, as therapeutic targets which capable of eliciting ADCC in tumor cells [8]. Recent findings were also exemplified the targeting of Cathepsin S in tumors, such as colorectal carcinomas, using antibody-based therapeutic inhibitors to block/inhibit tumor invasion and angiogenesis $[9,13]$. In conclusion, we hypothesize that the development of drug regimes that combine chemotherapies with an anti-invasive/antiangiogenic agent, such as the antibody described here, could lead to improved clinical outcomes in aggressive and metastatic tumors. Notably, elucidation of the humanized Cathepsin S inhibitory antibody implication in tumorigenesis may offer novel therapeutic options to complement currently available therapeutic strategies.

\section{ACKNOWLEDGEMENTS}

The Cathepsin S inhibitory antibody reported in this article was devel- oped by the Fusion Antibodies Ltd., UK.

\section{REFERENCES}

[1] Bania, J., Gatti, E., Lelouard, H., et al. (2003) Human cathepsin S, but not cathepsin L, degrades efficiently MHC class II-associated invariant chain in nonprofessional APCs. Proceedings of the National Academy of Sciences, 100, 6664-6669. doi:10.1073/pnas.1131604100

[2] Mohamed, M.M. and Sloane B.F. (2006) Cysteine cathepsins: Multifunctional enzymes in cancer. Nature Reviews, 6, 764-775. doi:10.1038/nrc1949

[3] Lindahl, C., Simonsson, M., Bergh, A., et al. (2009) Increased levels of macrophage-secreted cathepsin S during prostate cancer progression in TRAMP mice and patients. Cancer Genomics Proteomics, 6, 149-159.

[4] Paraoan, L., Gray, D., Hiscott, P., et al. (2009) Cathepsin $\mathrm{S}$ and its inhibitor cystatin C: Imbalance in uveal melanoma. Frontiers in Bioscience, 14, 2504-2513. doi: $10.2741 / 3393$

[5] Xu, J., Li, D., Ke, Z., et al. (2009) Cathepsin S is aberrantly overexpressed in human hepatocellular carcinoma. Molecular Medicine Reports, 2, 713-718.

[6] Flannery, T., McQuaid, S., McGoohan, C., et al. (2006) Cathepsin S expression: An independent prognostic factor in glioblastoma tumours-A pilot study. International Journal of Cancer, 15, 854-860. doi:10.1002/ijc.21911

[7] Gormley, J.A., Hegarty, S.M., O’Grady, A., et al. (2011) The role of Cathepsin $\mathrm{S}$ as a marker of prognosis and predictor of chemotherapy benefit in adjuvant CRC: A pilot study. British Journal of Cancer, 105, 1487-1494. doi:10.1038/bjc.2011.408

[8] Kwok, H.F., Buick, R.J., Kuehn, D., et al. (2011) Antibody targeting of Cathepsin S induces antibody-dependent cellular cytotoxicity. Molecular Cancer, 14, 147. doi:10.1186/1476-4598-10-147

[9] Burden, R.E., Gormley, J.A., Jaquin, T.J., et al. (2009) Antibody-mediated inhibition of cathepsin S blocks colorectal tumor invasion and angiogenesis. Clincial Cancer Research, 15, 6042-6051. doi:10.1158/1078-0432.CCR-09-1262

[10] Wang, B., Sun, J., Kitamoto, S., et al. (2006) Cathepsin S controls angiogenesis and tumor growth via matrix-derived angiogenic factors. Journal of Biological Chemistry, 281, 6020-6029. doi:10.1074/jbc.M509134200

[11] Ryschich, E., Lizdenis, P., Ittrich, C., et al. (2006) Molecular fingerprinting and autocrine growth regulation of endothelial cells in a murine model of hepatocellular carcinoma. Cancer Research, 66, 198-211. doi:10.1158/0008-5472.CAN-05-1636

[12] Gocheva, V., Zeng, W., Ke, D., et al. (2006) Distinct roles for cysteine cathepsin genes in multistage tumorigenesis. Genes \& Development, 20, 543-556. doi:10.1101/gad.1407406

[13] Ward, C., Kuehn, D., Burden, R.E., et al. (2010) Antibody targeting of cathepsin $\mathrm{S}$ inhibits angiogenesis and synergistically enhances anti-VEGF. PLoS One, 5, Arti- 
cle ID: e12543. doi:10.1371/journal.pone.0012543

[14] Burden, R.E., Gormley, J.A., Kuehn, D., et al. (2012) Inhibition of Cathepsin S by Fsn0503 enhances the efficacy of chemotherapy in colorectal carcinomas. Biochimie, 94, 487-493. doi:10.1016/j.biochi.2011.08.017

[15] Thurston, D.E. (2007) Chemistry and pharmacology of anticancer drugs. CRC Press (Taylor \& Francis), Florida.

[16] Schrama, D., Reisfeld, R.A. and Becker, J.C. (2006) Antibody targeted drugs as cancer therapeutics. Nature Re- views Drug Discovery, 5, 147-159. doi:10.1038/nrd1957

[17] Pastan, I., Hassan, R., Fitzgerald, D.J., et al. (2006) Immunotoxin therapy of cancer. Nature Reviews Cancer, 6, 559-565. doi:10.1038/nrc1891

[18] Zafir-Lavie, I., Michaeli, Y. and Reiter, Y. (2007) Novel antibodies as anticancer agents. Oncogene, 26, 3714-3733. doi:10.1038/sj.onc.1210372 